\title{
New detrital zircon U-Pb ages from BIF-related metasediments in the Ntem Complex (Congo craton) of southern Cameroon, West Africa
}

\author{
Nelson N. Chombong ${ }^{1 *}$, Emmanuel C. Suh ${ }^{1}$, Charles D. C. Ilouga ${ }^{2,3}$ \\ ${ }^{1}$ Economic Geology Unit, Department of Geology, University of Buea, Buea, Cameroon; \\ *Corresponding Author: nchombong@yahoo.com \\ ${ }^{2}$ Department of Earth Sciences, University of Yaoundé I, Yaoundé, Cameroon \\ ${ }^{3}$ The Cameroon Iron Ore Company (CamIron S.A.), Yaoundé, Cameroon
}

Received 30 March 2013; revised 2 May 2013; accepted 10 May 2013

Copyright (C) 2013 Nelson N. Chombong et al. This is an open access article distributed under the Creative Commons Attribution License, which permits unrestricted use, distribution, and reproduction in any medium, provided the original work is properly cited.

\begin{abstract}
Banded Iron Formations (BIFs) were formed by contemporaneous events of active sediments supply and the venting of a hydrothermal fluid source at the Mid-Ocean-Ridge. BIFs within the Ntem Complex at the northern edge of the Congo Craton are intercalated with metasandstones and metasiltstones. SHRIMP U-Pb analysis on detrital zircons obtained from a metasandstone gave variable ages from over $3000 \mathrm{Ma}$ to $1000 \mathrm{Ma}$ with the maximum age of deposition clustered around $1200 \mathrm{Ma}$ and the peak of deposition at $1800 \mathrm{Ma}$. This age range suggested that the sub-basin was opened sometime in the Archean and remained active up till the Neoproterozoic. Zircons with Archean ages have a provenance linked to the charnockitic suite and the high-K granites within the Ntem Complex. The Paleoproterozoic ages are attributed to clastic inputs from the neigbouring Nyong Series west of the Ntem Complex. Also the peak of deposition in the Proterozoic could probably be explained by the globally recognized intense crust-forming processes in the Early Proterozoic time. The provenance of the younger Neoproterozoic ages is tied to various lithologies within the northern mobile belts of the Adamawa-Yade massifs and correlates with Neoproterozoic sedimentation ages in the Yaoundé, Lom and Poli series. The Neoproterozoic ages are comparable to those obtained from metasediments of the Amazonian Craton and provide evidence of Pre-Gondwana assemblage of the Congo and the São Francisco Cratons.
\end{abstract}

Keywords: BIFs; Metasediments; Ntem Complex; SHRIMP U-Pb Age; Detrital Zircons

\section{INTRODUCTION}

Banded Iron Formations (BIFs) consist of alternating Si-rich and Fe-rich layers [1,2]. BIFs are formed through two simultaneous processes: clastic sediment supply from the weathering of continental masses and submarine volcanism and associated hydrothermal activity at the MidOcean-Ridge (MOR) [3-9]. The classical nature of this enigmatic rock type around the world today suggests an extraordinary record of $\mathrm{Fe}$ and $\mathrm{Si}$ cycles in the earth's history which is still poorly understood [10]. It is widely believed that the source of both the Fe and Si in the BIFs is largely from the ocean. However, some authors have shown that the contribution of iron and silica through clastic inputs from the continent is also eminent in the formation of the thick BIF sequences observed around the world. Voluminous tholeiitic basalts extrusion rich in Fe have been reported from mantle plume events [11]. According to [12], pre-erupted continental flood basalts from such an event through the rifting of the continental crust $[13,14]$ are subsequently eroded and increase riverine flux of $\mathrm{Fe}$ to the ocean. In addition, isotopic studies have reported $\delta^{30} \mathrm{Si}$ values in BIFs which reflect elevated temperatures of seawater and the influence of a continental source [15-17]. Recently, [9] has also described textural characteristics such as the allochems in iron formations which are largely granules (clastics) and point to a continental input while the matrix which is mostly chemical iron-rich muds and microcrystalline quartz (chert) is precipitated from the ocean. $[8,18]$ have earlier identified these granules as being endoclastic in origin and derived from the re-working of earlier lithified Fe-for- 
mation components. These arguments in favour of Si and Fe derived from the continent warrant a succinct understanding of the age and nature of the provenance areas during the formation of BIFs. In the Ntem Complex (Congo craton) BIFs are associated with metasediments that have not been investigated although they can provide information on the evolution of the basin and age of the rocks from which the sediments were derived. Though metasedimentary and metamorphic siliciclastic rocks have been given little attention in literature, their usefulness in geochronological studies is vital as they often provide constraints on the possible composition, tectonic setting, palaeoweathering and the evolution of the early upper continental crust. This study therefore investigates the age of BIF-related metasediments within the Ntem complex with the aim of deciphering the period of deposition and the basin evolutionary history in comparison with other major iron ore basins across the Atlantic.

\section{GEOLOGICAL REVIEW}

The Ntem Complex is part of the Congo craton and represents the oldest lithologic group in Cameroon. This complex hosts several iron deposits at various stages of resource definition and development (see [23-25,39] and www.sundanceresources.com.au, www.afferro-mining.com).

Tonalite-trondhjemite-granodiorite [19,20], greenstones and metasedimentary formations define the basement rock suit of the Ntem Complex [21,22]. These are intruded by much younger Late high-K granites and dolerite dykes [20,22] (see Figure 1 below).

These are thought to have been emplaced at the same time and are interpreted as a heat source for remelting TTG and charnockites [20,22]. Monzogranites and syenogranites represent the high-K granitoids pods and tongues occurring with mafic restite and small scale pegmatitic and aplitic veins and dykes [20]. Rock units of economic importance in the Ntem Complex include high grade iron ore and itabirites [23-26] with less prominent ultrabasic rocks containing elevated $\mathrm{Ni}, \mathrm{Cr}$ and Co contents [27].

Two major deformations have been recognized at the northwestern edge of the Congo craton [28]. A non-rotational D1 that defines the S1 foliation developed during crystallization [29]. D2 defines the deformation of the $S_{1}$ foliation into low amplitude folds oriented N80E to N120E and N-S [29] accompanied by the emplacement of plutons, diapirism and recrystallization. This is evident from relict greenstone belts and the TTG series [30]. Wide spread $\mathrm{C}_{2}$ surfaces associated with partial melting of the TTG and greenstones belt [30] define sinistral shear planes that trend N-S to N45E-N50E. High dip $\mathrm{C}_{3}$ mylonitic planes and shear corridors observed at the contact with the Yaoundé nappe front $[19,30]$ define D3 and D4.

The samples investigated in this study were derived from the Njweng prospect within the Mbalam iron ore district (Figure 2). At Njweng the main rock types are BIFs, metagranitoids and amphibolites associated with metasediments [26]. On the surface, the BIFs are partially altered with variable magnetic intensity and hematite and goethite content [26].

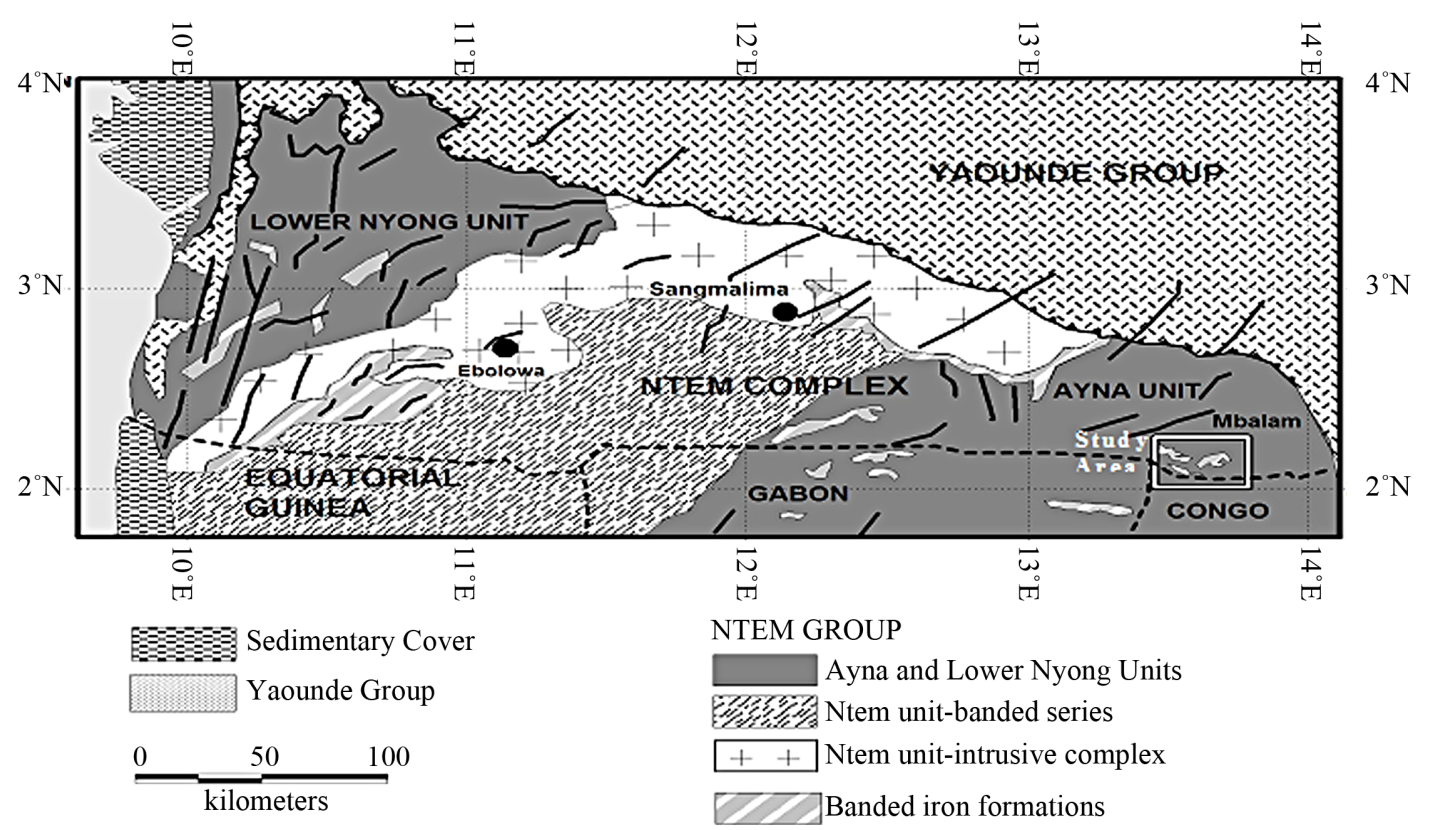

Figure 1. Regional geological map of the northern limit of the Archaean Congo craton (Ntem complex, the Nyong Unit) showing the Mbalam BIF units (study area blocked in white rectangle on map), late Archean granitic intrusions, and thrust contact with the Pan-African fold belt in Southern Cameroon. Modified after [67]. 


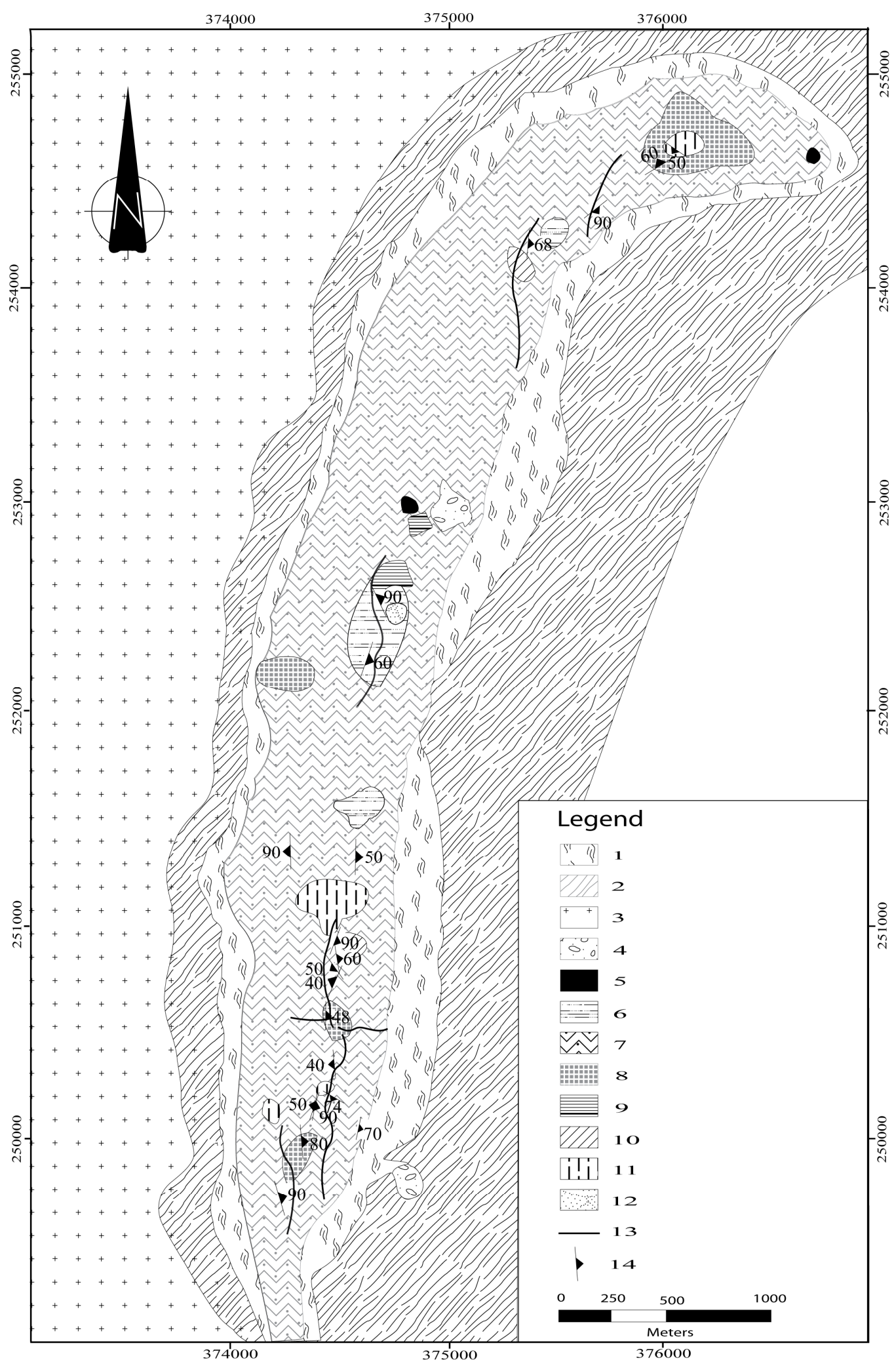

Figure 2. Geological sketch of the Njweng prospect mapped for the first time using combined aeromagnetic data and ground-truthing. 1: amphibolite; 2: Garnet amphibolite; 3: Metagranitoids; 4: Breccia/Conglomerate BIF; 5: Martite-Goethite; 6: Granular and Massive bands BIF; 7: Granular bands BIF; 8: Massive bands BIF; 9: Weakly banded BIF; 10: Specularite bands BIF; 11: Amphibolitic bands BIF (silicate facies); 12: Metasandstone; 13: Fault; 14: Strike and Dip of prominent foliation trends. 
$\mathrm{U}-\mathrm{Pb}$ analysis on zircons, $\mathrm{Sm}-\mathrm{Nd}$ and $\mathrm{Pb}-\mathrm{Pb}$ evaporation ages on whole rock and other geochronological data have confirmed the Archean to Paleoproterozoic age of the Congo and the São Francisco Cratons [19,31-36]. However, Mesoproterozoic to early Neoproterozoic (1100 - $910 \mathrm{Ma}$ ) ages of magmatic and sedimentary sequences have been reported at the northwestern edge of the Congo Craton. This age margin is largely tied to the PanAfrican-Braziliano Yaoundé and Adamawa-Yadé and the Sergipano domains north of the Congo and São Francisco cratons, respectively. A minimum age of $628 \pm 12$ Ma has been reported [37] to constraint the age of the Pan-African-Braziliano orogeny. A similar age of $626 \mathrm{Ma}$ has been reported on detrital zircons from the Yaoundé micaschists [38]. The possibility of clastics from these younger basins being supplied during BIF deposition in the Congo Craton (Ntem Complex) is yet to be examined. In this paper we characterize metasediments associated with BIF in the Ntem complex and date detrital zircons from them in order to unravel their provenance and the palaeodepositional environment. The mineralogy, age and the structural setting of the BIF have been reported [23-25,39].

\section{Samples Description, Preparation and Analytical Procedure}

Two metasedimenatary rock samples associated with BIFs at the Njweng Prospect, Mbalam Iron ore deposit, in the Ntem Complex were investigated in terms of their petrography and terms of their petrography and whole rock geochemistry with one of the sample eventually dated. The samples were a metasiltstone (NTM10) and a metasandstone (NTM194). Sample NTM10 was cream white in color and very finegrained while NTM194 was reddish brown in color and fine-grained. Sample NTM194 was dated by SHRIMP U-Pb on separated zircons to provide geochronology data from which the sediments provenance and the paleodepositional evolution of the basin in relation to BIF formation can be deduced.

Major and trace elements compositions of the samples were obtained by ICP-OES and ICP-MS at a commercial laboratory (Acme Analytical Laboratories Ltd., Vancouver, Canada) and the details are provided in [39].

Zircon separation was performed in the laboratories of the Research School of Earth Sciences (RSES), Australian National University (ANU). The sample was crushed and milled, and the fines washed off in a settling beaker. Magnetic minerals were separated using a hand magnet and a Franz isodynamic separator. Heavy minerals were concentrated using both tetrabromoethane and methylene iodide. Concentrated zircons were mounted in epoxy, together with Temora III $(416.8 \pm 1.3 \mathrm{Ma}$; [40], FC1 (1099.1 $\pm 0.5 \mathrm{Ma}$; [41] and SL13 reference zircons. Te- mora is the primary $\mathrm{U}-\mathrm{Pb}$ standard, $\mathrm{FC} 1$ was used as a secondary standard and to monitor ${ }^{207} \mathrm{~Pb} /{ }^{206} \mathrm{~Pb}$ ratios, and SL13 [42] is a chip of a single crystal with a uniform $U$ content and is used to calibrate $\mathrm{U}, \mathrm{Th}$ and $\mathrm{Pb}$ concentration.

Analyses of the zircon grains were also done at the RSES and the procedure is recorded in [39]]. The data were processed using the SQUID I Excel Macro of [43, $44]$ and the decay constants of [45] were employed. Uncertainties in the calculated weighted mean ages are reported as $95 \%$ confidence limits. For the age calculations, corrections for common $\mathrm{Pb}$ were made using the measured ${ }^{204} \mathrm{~Pb}$ and the relevant common $\mathrm{Pb}$ compositions from the [46] model while the concordia plots were done with Isoplot/Ex 3.0 [47].

\section{RESULTS}

\subsection{Petrography}

The metasandstone has quartz grains that are elongated, angular to sub-rounded with grain boundaries of $120^{\circ}$ typical of recrystallized quartz (Figure 3(a)). The rock has a clast supported fabric dominated by quartz grains with $<5 \%$ matrix defining an epiclastic texture eminent in an arenite (see Figures 3(a) and (b)). The poor matrix content of the rock is indicative of its high maturity. Disseminated sulfides are observed in the iron-oxide matrix. The elongated quartz grains also show a preferred orientation indicative of recrystallization under directed stress (Figure 3(b)).

The metasiltstone on the other hand shows a strong diagenetic foliation defined by distinct sub-parallel quartzrich bands that are oblique to mica-rich bands (Figure 3(c)). This is typical in low grade pelitic sediments with detrital micas that have undergone little or no deformation [48]. The rock is poorly sorted with porphyroblasts of muscovite observed within a quartz-rich groundmass (Figure 3(d)). This is also indicative of the immature nature of the sediments. The slightly oblique nature of the muscovite porphyroblasts to the general foliation in the rock is indicative of recrystallization under directed stress (see Figure 3(e)). This is also expressed by the development of weak micro kink-bands on some of the muscovite porphyroblasts (Figure 3(e)) in which quartz inclusions are ubiquitous (Figure 3(e)).

\subsection{Whole Rock Geochemistry}

Major and trace element data of the 2 samples analyzed are listed in Table 1. The sandstone and siltstone have $\mathrm{SiO}_{2}$ contents of 95.26 and $73.74 \mathrm{wt} \%, \mathrm{Al}_{2} \mathrm{O}_{3}, 1.48$ and $14.86 \mathrm{wt} \%$ and $\mathrm{MgO}, 0.06$ and $1.82 \mathrm{wt} \%$, respectively. These values are similar to sand and silt values of sea sediments [49] of the Paleoproterozoic age [50]. 

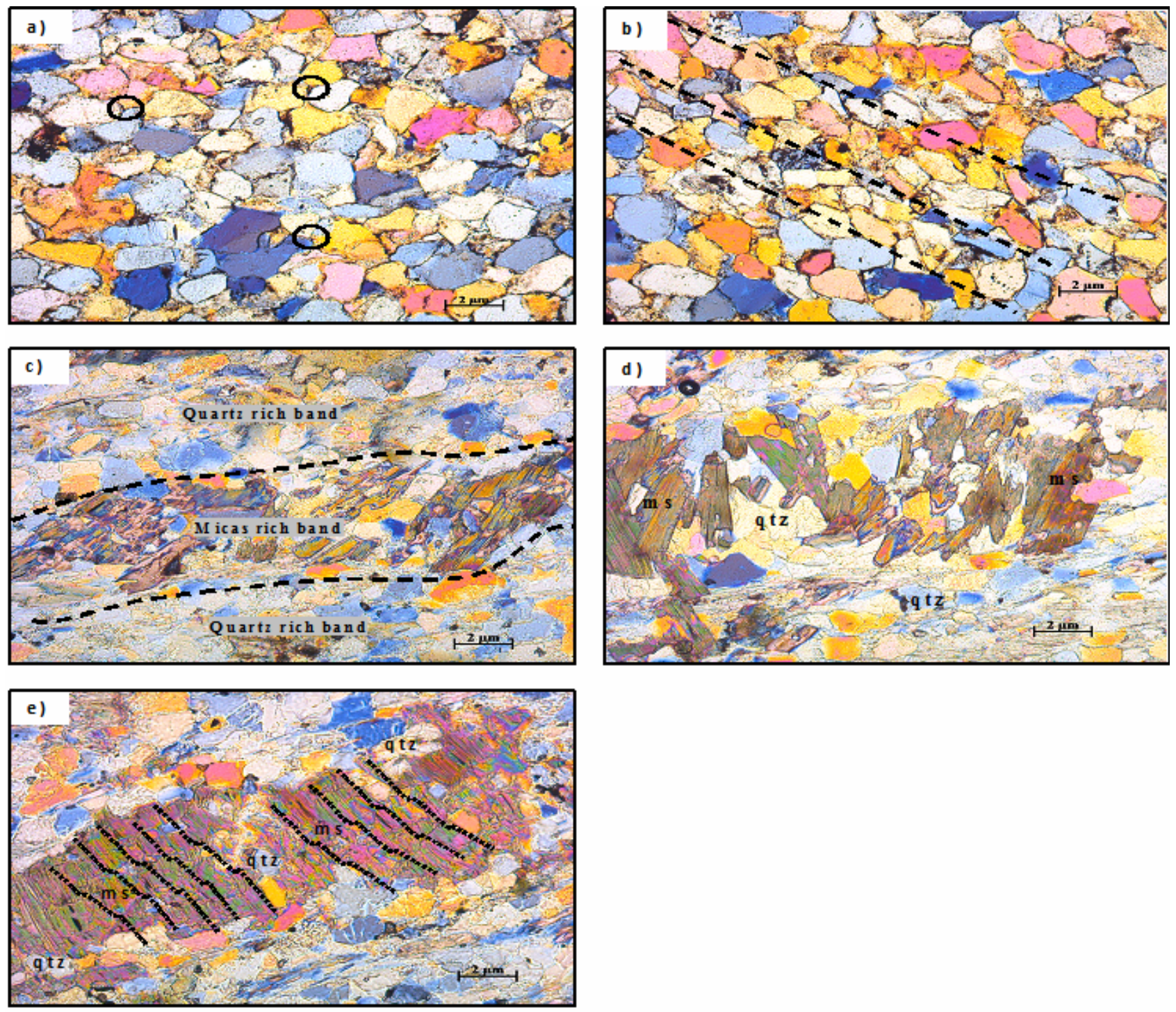

Figure 3. (a) Metasandstone with elongated, angular and sub-rounded quartz grains. Black circles show $120^{\circ}$ angle between quartz grains. Rock is clast supported defining an epiclastic texture; (b) Metasandstone. Note that elongated quartz grains define a preferred orientation due to recrystallization under directed stress. Black lines show the orientation trend; (c) Metasiltstone with a strong foliation defined by distinct bands of quartz and micas. Also note the oblique orientation of the micas to the general foliation trend of the rock; (d) Metasiltstone showing muscovite porphyroblast within a quartz-rich groundmass. "ms" = muscovite and "qtz" = quartz; (e) Metasiltstone showing weak micro kink-bands developed on a muscovite (ms) porphyroblast due to recrystallization under directed stress. Also note quartz (qtz) inclusions within the muscovite porphyroblast.

However, the sandstone has a slightly elevated $\mathrm{SiO}_{2}$ content which is probably attributed to its high quartz content owing to the resistance of quartz during transportation. Also, $\mathrm{CaO}$ and $\mathrm{Na}_{2} \mathrm{O}$ values of $0.02,0.11 \mathrm{wt} \%$ and $0.02,0.30 \mathrm{wt} \%$ of the metasandstone and metasiltstone, respectively, are close to values obtained for shales of early Archean greenstone belts [51]. These variations suggest different provenance for sediments into the ba$\sin$.

A plot of $\mathrm{K}_{2} \mathrm{O} / \mathrm{Na}_{2} \mathrm{O}$ against $\mathrm{SiO}_{2} / \mathrm{Al}_{2} \mathrm{O}_{3}$ for the samples reflects Post Archean Australian greenstone Shales values obtained by Taylor \& McLennan (1985) with
$\mathrm{K}_{2} \mathrm{O} / \mathrm{Na}_{2} \mathrm{O}>1$. The wide difference between the $\mathrm{SiO}_{2} /$ $\mathrm{Al}_{2} \mathrm{O}_{3}$ value of the metasandstone (64.36) compared to the metasiltstone (4.96) mainly confirms the maturity of the metasandstone [52].

Post Archean Australian shale (PAAS) normalized values of the rare earth elements (REEs) data of the metasandstone and metasiltstone show a relatively flat pattern with values generally $<1$ typical of continental sediments $[53,54]$ (see Figure 4). This is also expressed by the $(\mathrm{La} /$ $\mathrm{Yb}) \mathrm{N}$ values that are $<1$. Ce shows a flat pattern in both the metasandstone and metasiltstone while Eu expresses a flat and weak negative anomaly in the metasandstone 


\begin{tabular}{|c|c|c|c|c|c|c|c|}
\hline$\vec{N}$ & 言 & $\bar{\infty}$ & $\begin{array}{l}\overrightarrow{8} \\
i\end{array}$ & & ஜ & 言 & $\ddot{\theta}$ \\
\hline 3 & है & $\tilde{\hat{v}}$ & $\Xi$ & & $F$ & ह & $\overrightarrow{\dot{\vec{v}}}$ \\
\hline$>$ & 袬 & $=$ & $a$ & & $\stackrel{000}{I}$ & 言 & $\overrightarrow{0}$ \\
\hline D & ह⿳亠二口犬 : & $\dot{o}$ & $\stackrel{m}{m}$ & & $\bar{\psi}$ & 言 & $\tilde{v}_{\hat{v}}^{n}$ \\
\hline$\Xi$ & हً & $\stackrel{n}{i}$ & 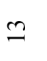 & & $\stackrel{80}{<}$ & 言 & $\overrightarrow{\dot{\vec{v}}}$ \\
\hline$\approx$ & हू & 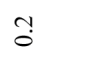 & $\Xi$ & & $\bar{\infty}$ & 竐 & $\overrightarrow{\vec{v}}$ \\
\hline$\dot{n}$ & 言 & $\begin{array}{l}0 \\
\infty\end{array}$ & $\simeq$ & & क & 言 & $\overrightarrow{\dot{\vec{v}}}$ \\
\hline हี & 흘 & $\bar{v}$ & in & & $\vec{J}$ & 言 & $\overline{\dot{\vec{v}}}$ \\
\hline थิ & हิ & $\underbrace{\infty}_{0}$ & 良 & & \& & 赔 & - \\
\hline$\vec{z}$ & 言 & $\ddot{r}$ & $\stackrel{\dot{\Xi}}{\dot{S}}$ & & $\bar{z}$ & 言 & $\stackrel{0}{\circ}$ \\
\hline$\Psi$ & 言 & $\stackrel{\infty}{\sim}$ & in & & $\Xi$ & పิ & $\vec{v}$ \\
\hline$\tilde{0}$ & 言 & $\stackrel{\circ}{-}$ & $\bar{\infty}$ & & 20 & 言 & - \\
\hline 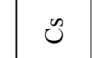 & 言 & ֻุ & $\stackrel{\dot{m}}{\dot{m}}$ & & $\vec{U}$ & 言 & $n$ \\
\hline 8 & हू & 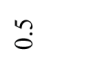 & $\stackrel{\infty}{-}$ & & $\sum^{\circ}$ & 言 & $\overline{\dot{\hat{v}}}$ \\
\hline 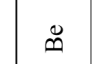 & हิ & $\bar{v}$ & $\bar{v}$ & & $\frac{\omega}{6}$ & $\therefore$ & $\stackrel{\check{\delta}}{\dot{\delta}}$ \\
\hline$\cong \widetilde{n}$ & 틀 & q & $\underset{\infty}{\mathbb{\infty}}$ & & $\stackrel{\cup}{E}$ & $\therefore$ & $\stackrel{8}{0}$ \\
\hline in & Е & - & in & 气ूँ & $\exists$ & छํㅡㄹ & $\stackrel{0}{\div}$ \\
\hline$\vec{z}$ & हิ & $\stackrel{\nabla}{\vee}$ & ชิ & & $\stackrel{\lambda}{2}$ & ह & $\tilde{\alpha}$ \\
\hline 悫 & $\alpha^{\circ}$ & $\partial$ & $\infty$ & & $\Xi$ & ह & $\stackrel{m}{=}$ \\
\hline o & $d^{\circ}$ & $\stackrel{\infty}{0}$ & 恶 & & 画 & ह & $\underset{\infty}{\infty}$ \\
\hline 0 & $\delta^{\circ}$ & $\begin{array}{l}\text { oे } \\
\text {. }\end{array}$ & ठ্ণ & & $\stackrel{\circ}{1}$ & ह & ֻั̊ \\
\hline$\stackrel{\circ}{g}$ & $\AA^{\circ}$ & $\bar{\sigma}$ & $\bar{o}$. & & $\widehat{a}$ & ह & $\bar{\uparrow}$ \\
\hline $\begin{array}{l}4 \\
0 \\
0\end{array}$ & 0 & & $\delta$ & & 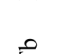 & $\Xi$ & ฯ \\
\hline$\Omega^{2}$ & 8 & 0 & 0 & & $F$ & בे & 0 \\
\hline 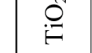 & $\dot{0}^{\circ}$ & $\frac{n}{0}$ & $\frac{m}{0}$ & & $\bar{J}$ & 흘 & $\stackrel{\varrho}{\leftrightarrows}$ \\
\hline$\stackrel{0}{2}$ & $\therefore$ & $\bar{m}$ & $\stackrel{\infty}{\stackrel{\infty}{\infty}}$ & & $\vec{w}$ & 言 & ֶิ \\
\hline $\begin{array}{l}0 \\
\text { है }\end{array}$ & $\circ^{\circ}$ & 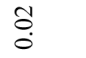 & ?3 & & ह & 言 & $\stackrel{n}{=}$ \\
\hline 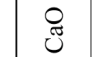 & $a^{\circ}$ & $\stackrel{1}{0}$ & $\overline{\overline{0}}$ & & $\bar{z}$ & E⿳亠二口⿱幺小 & $\hat{\sigma}$ \\
\hline $\begin{array}{l}\frac{O}{00} \\
\frac{a n}{2}\end{array}$ & $0^{\circ}$ & $\stackrel{\circ}{\circ}$ & ه్ & & 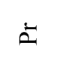 & ह & $\overrightarrow{\bar{i}}$ \\
\hline$\overbrace{}^{\infty}$ & $a^{\circ}$ & $\stackrel{\infty}{-\infty}$ & 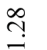 & & $\ddot{0}$ & 言 & $\stackrel{\infty}{\stackrel{\sim}{0}}$ \\
\hline $\begin{array}{l}0 \\
\stackrel{1}{<}\end{array}$ & $\circ^{\circ}$ & $\stackrel{\infty}{\stackrel{\infty}{-}}$ & 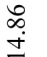 & & $\Xi$ & 言 & $\stackrel{\tilde{o}}{0}$ \\
\hline$\tilde{n}^{N}$ & $\dot{0}^{\circ}$ & $\begin{array}{l}\text { त् } \\
\text { ñ }\end{array}$ & $\stackrel{d}{d}$ & & $>$ & 흠 & $\underset{\infty}{+}$ \\
\hline$\delta$ & 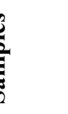 & $\sum_{\mathrm{z}}^{\mathrm{J}}$ & $\sum_{z}^{O}$ & & & & $\sum_{\bar{z}}^{\stackrel{J}{E}}$ \\
\hline
\end{tabular}


Table 2. Summary of new SHRIMP U-Pb detrital zircon data for sample NTM194.

\begin{tabular}{|c|c|c|c|c|c|c|c|c|c|c|c|c|c|c|c|}
\hline $\begin{array}{c}\text { Grain. } \\
\text { Spot }\end{array}$ & $\begin{array}{c}\% \\
{ }^{206} \mathbf{P b}_{\mathrm{c}}\end{array}$ & $\underset{\mathbf{U}}{\mathbf{p p m}}$ & $\begin{array}{l}\text { ppm } \\
\text { Th }\end{array}$ & $\begin{array}{l}{ }^{232} \mathrm{Th} / \\
{ }^{238} \mathrm{U}\end{array}$ & $\underset{{ }^{206} \mathbf{P b}^{*}}{\mathbf{p p m}}$ & $\begin{array}{c}(1) \\
{ }^{206} \mathrm{~Pb}^{238} \mathrm{U} \\
\text { Age }\end{array}$ & $\begin{array}{c}(1) \\
{ }^{207} \mathbf{P b} /{ }^{206} \mathrm{~Pb} \\
\text { Age }\end{array}$ & $\begin{array}{c}\% \\
\text { Discordant }\end{array}$ & $\underset{{ }^{207} \mathbf{P b}^{*} /{ }^{206} \mathbf{P b}^{*}}{ }$ & $\pm \%$ & $\stackrel{(1)}{{ }^{207} \mathbf{P b}^{*} /{ }^{235} \mathbf{U}}$ & $\pm \%$ & $\begin{array}{c}(\mathbf{1}) \\
{ }^{206} \mathbf{P b}^{*} \\
2^{238} \mathbf{U}\end{array}$ & $\pm \%$ & $\begin{array}{l}\text { err } \\
\text { corr }\end{array}$ \\
\hline 1.1 & 0.09 & 250 & 179 & 0.74 & 66.9 & $1747 \pm 17$ & $1770.3 \pm 9.2$ & 1 & 0.10826 & 0.51 & 4.647 & 1.2 & 0.3113 & 1.1 & 0.914 \\
\hline 2.1 & 0.93 & 43 & 47 & 1.11 & 7.98 & $1240 \pm 20$ & $1244 \pm 160$ & 0 & 0.0819 & 8.3 & 2.4 & 8.5 & 0.2121 & 1.7 & 0.204 \\
\hline 3.1 & 0.34 & 541 & 134 & 0.26 & 104 & $1302 \pm 13$ & $1390 \pm 12$ & 6 & 0.08832 & 0.64 & 2.726 & 1.2 & 0.2238 & 1.1 & 0.858 \\
\hline 4.1 & 0.12 & 272 & 185 & 0.70 & 47.1 & $1183 \pm 12$ & $1193 \pm 16$ & 1 & 0.07983 & 0.83 & 2.216 & 1.4 & 0.2014 & 1.1 & 0.809 \\
\hline 5.1 & 0.00 & 288 & 98 & 0.35 & 69.9 & $1602 \pm 16$ & $1567 \pm 12$ & -2 & 0.097 & 0.65 & 3.774 & 1.3 & 0.2822 & 1.1 & 0.866 \\
\hline 6.1 & 0.02 & 283 & 130 & 0.47 & 64.6 & $1518 \pm 16$ & $1513 \pm 10$ & 0 & 0.09425 & 0.55 & 3.45 & 1.3 & 0.2655 & 1.2 & 0.902 \\
\hline 7.1 & 0.08 & 144 & 78 & 0.56 & 39 & $1771 \pm 20$ & $1751 \pm 13$ & -1 & 0.10714 & 0.73 & 4.672 & 1.5 & 0.3162 & 1.3 & 0.866 \\
\hline 8.1 & 0.00 & 151 & 169 & 1.15 & 42.2 & $1813 \pm 20$ & $1794 \pm 17$ & -1 & 0.1097 & 0.94 & 4.91 & 1.6 & 0.3247 & 1.3 & 0.805 \\
\hline 9.1 & 0.10 & 151 & 81 & 0.55 & 40.4 & $1745 \pm 20$ & $1768 \pm 13$ & 1 & 0.10811 & 0.72 & 4.634 & 1.5 & 0.3109 & 1.3 & 0.873 \\
\hline 10.1 & 0.16 & 358 & 467 & 1.35 & 87.4 & $1608 \pm 16$ & $1767 \pm 10$ & 9 & 0.10809 & 0.56 & 4.223 & 1.3 & 0.2834 & 1.1 & 0.893 \\
\hline 11.1 & 0.12 & 289 & 137 & 0.49 & 84 & $1879 \pm 19$ & $2097.3 \pm 8.4$ & 10 & 0.12995 & 0.48 & 6.062 & 1.2 & 0.3383 & 1.1 & 0.922 \\
\hline 12.1 & 0.07 & 143 & 139 & 1.00 & 45.8 & $2037 \pm 23$ & $2108 \pm 13$ & 3 & 0.13073 & 0.71 & 6.698 & 1.5 & 0.3716 & 1.3 & 0.876 \\
\hline 13.1 & 0.10 & 258 & 103 & 0.41 & 49.9 & $1305 \pm 14$ & $1231 \pm 16$ & -6 & 0.08141 & 0.79 & 2.519 & 1.4 & 0.2244 & 1.2 & 0.826 \\
\hline 14.1 & 2.45 & 123 & 88 & 0.74 & 22.7 & $1230 \pm 16$ & $1245 \pm 73$ & 1 & 0.082 & 3.7 & 2.376 & 4 & 0.2102 & 1.4 & 0.348 \\
\hline 15.1 & 0.00 & 212 & 109 & 0.53 & 51.5 & $1605 \pm 17$ & $1570 \pm 11$ & -2 & 0.09715 & 0.6 & 3.787 & 1.3 & 0.2827 & 1.2 & 0.893 \\
\hline 16.1 & 0.10 & 211 & 92 & 0.45 & 42.1 & $1344 \pm 15$ & $1329 \pm 15$ & -1 & 0.08561 & 0.8 & 2.737 & 1.4 & 0.2319 & 1.2 & 0.833 \\
\hline 17.1 & 0.04 & 164 & 72 & 0.45 & 44.3 & $1765 \pm 19$ & $1768 \pm 13$ & 0 & 0.1081 & 0.7 & 4.694 & 1.4 & 0.3149 & 1.3 & 0.874 \\
\hline 18.1 & 1.16 & 753 & 1112 & 1.52 & 112 & $1019 \pm 10$ & $1717 \pm 19$ & 41 & 0.1052 & 1 & 2.483 & 1.5 & 0.1712 & 1.1 & 0.721 \\
\hline 19.1 & 0.00 & 87 & 93 & 1.10 & 25.8 & $1916 \pm 24$ & $1888 \pm 14$ & -1 & 0.11554 & 0.79 & 5.514 & 1.6 & 0.3461 & 1.4 & 0.876 \\
\hline 20.1 & 0.00 & 222 & 74 & 0.35 & 48.2 & $1449 \pm 15$ & $1424 \pm 12$ & -2 & 0.08994 & 0.64 & 3.125 & 1.4 & 0.252 & 1.2 & 0.881 \\
\hline 21.1 & 0.05 & 128 & 194 & 1.56 & 44.7 & $2198 \pm 24$ & $2203.9 \pm 9.9$ & 0 & 0.13813 & 0.57 & 7.74 & 1.4 & 0.4064 & 1.3 & 0.916 \\
\hline 22.1 & 0.04 & 128 & 100 & 0.81 & 29.3 & $1522 \pm 18$ & $1525 \pm 16$ & 0 & 0.09485 & 0.87 & 3.483 & 1.6 & 0.2663 & 1.3 & 0.839 \\
\hline 23.1 & 0.00 & 96 & 67 & 0.72 & 29.5 & $1963 \pm 24$ & $1958 \pm 14$ & 0 & 0.12009 & 0.76 & 5.895 & 1.6 & 0.356 & 1.4 & 0.879 \\
\hline 24.1 & 0.03 & 273 & 317 & 1.20 & 76.1 & $1812 \pm 18$ & $1878.4 \pm 8.4$ & 4 & 0.1149 & 0.46 & 5.142 & 1.2 & 0.3245 & 1.1 & 0.927 \\
\hline 25.1 & 1.18 & 882 & 436 & 0.51 & 116 & $910.6 \pm 9$ & $1246 \pm 25$ & 27 & 0.082 & 1.3 & 1.716 & 1.7 & 0.1517 & 1.1 & 0.638 \\
\hline 26.1 & 0.06 & 151 & 96 & 0.66 & 40.6 & $1755 \pm 20$ & $1811 \pm 12$ & 3 & 0.11071 & 0.67 & 4.778 & 1.4 & 0.313 & 1.3 & 0.883 \\
\hline 27.1 & 0.16 & 553 & 86 & 0.16 & 130 & $1554 \pm 15$ & $1717.7 \pm 8.6$ & 10 & 0.10519 & 0.47 & 3.955 & 1.2 & 0.2727 & 1.1 & 0.918 \\
\hline 28.1 & 0.60 & 480 & 278 & 0.60 & 70.2 & $1007 \pm 10$ & $1449 \pm 18$ & 30 & 0.09109 & 0.96 & 2.123 & 1.5 & 0.169 & 1.1 & 0.755 \\
\hline 29.1 & 0.14 & 280 & 222 & 0.82 & 75.6 & $1761 \pm 18$ & $1934 \pm 9.8$ & 9 & 0.11851 & 0.55 & 5.132 & 1.3 & 0.314 & 1.1 & 0.903 \\
\hline 30.1 & 0.08 & 112 & 60 & 0.55 & 24.8 & $1480 \pm 19$ & $1508 \pm 20$ & 2 & 0.09399 & 1.1 & 3.344 & 1.8 & 0.2581 & 1.4 & 0.803 \\
\hline 31.1 & 0.09 & 340 & 104 & 0.32 & 64.7 & $1289 \pm 14$ & $1420 \pm 16$ & 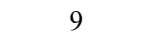 & 0.08975 & 0.84 & 2.74 & 1.5 & 0.2214 & 1.2 & 0.825 \\
\hline 32.1 & 0.09 & 280 & 68 & 0.25 & 50.4 & $1225 \pm 14$ & $1362 \pm 19$ & 10 & 0.08705 & 0.98 & 2.513 & 1.6 & 0.2094 & 1.3 & 0.790 \\
\hline 33.1 & 0.09 & 117 & 92 & 0.81 & 34.6 & $1903 \pm 24$ & $1980 \pm 17$ & 4 & 0.1216 & 0.93 & 5.76 & 1.7 & 0.3435 & 1.4 & 0.838 \\
\hline 34.1 & 0.03 & 479 & 301 & 0.65 & 82.8 & $1182 \pm 12$ & $1223 \pm 12$ & 3 & 0.08107 & 0.6 & 2.249 & 1.3 & 0.2012 & 1.1 & 0.880 \\
\hline 35.1 & 0.02 & 247 & 95 & 0.40 & 82.9 & $2124 \pm 21$ & $2128.6 \pm 7.3$ & 0 & 0.13229 & 0.42 & 7.118 & 1.2 & 0.3903 & 1.2 & 0.941 \\
\hline 36.1 & 0.02 & 126 & 81 & 0.66 & 42 & $2113 \pm 24$ & $2107 \pm 11$ & 0 & 0.13068 & 0.61 & 6.99 & 1.4 & 0.3879 & 1.3 & 0.908 \\
\hline 37.1 & 0.25 & 284 & 221 & 0.81 & 112 & $2432 \pm 25$ & $2532.6 \pm 7.9$ & 4 & 0.16748 & 0.47 & 10.59 & 1.3 & 0.4584 & 1.2 & 0.935 \\
\hline 38.1 & 0.00 & 80 & 119 & 1.53 & 37 & $2773 \pm 35$ & $2714 \pm 10$ & -2 & 0.1867 & 0.64 & 13.84 & 1.7 & 0.5375 & 1.5 & 0.925 \\
\hline 39.1 & - & 192 & 120 & 0.65 & 61.3 & $2036 \pm 21$ & $2059.7 \pm 9.4$ & 1 & 0.12721 & 0.53 & 6.514 & 1.3 & 0.3714 & 1.2 & 0.918 \\
\hline 40.1 & 0.16 & 149 & 77 & 0.54 & 33.7 & $1507 \pm 18$ & $1507 \pm 19$ & 0 & 0.09392 & 10 & 3.411 & 1.6 & 0.2634 & 1.3 & 0.797 \\
\hline 41.1 & 0.05 & 91 & 55 & 0.62 & 20.9 & $1534 \pm 19$ & $1528 \pm 19$ & 0 & 0.09501 & 1 & 3.52 & 1.7 & 0.2687 & 1.4 & 0.815 \\
\hline 42.1 & 0.06 & 180 & 130 & 0.75 & 47.7 & $1733 \pm 19$ & $1746 \pm 12$ & 1 & 0.1068 & 0.66 & 4.542 & 1.4 & 0.3084 & 1.2 & 0.884 \\
\hline 43.1 & 0.05 & 181 & 155 & 0.89 & 49.4 & $1776 \pm 19$ & $1796 \pm 11$ & 1 & 0.10979 & 0.62 & 4.803 & 1.4 & 0.3173 & 1.2 & 0.894 \\
\hline 44.1 & 0.13 & 144 & 130 & 0.93 & 60.1 & $2551 \pm 27$ & $3024 \pm 10$ & 16 & 0.2261 & 0.64 & 15.13 & 1.4 & 0.4855 & 1.3 & 0.898 \\
\hline 45.1 & 0.24 & 94 & 102 & 1.12 & 24.1 & $1682 \pm 23$ & $1889 \pm 20$ & 11 & 0.1156 & 1.1 & 4.751 & 1.9 & 0.2982 & 1.6 & 0.814 \\
\hline 46.1 & 0.19 & 237 & 139 & 0.61 & 39.3 & $1135 \pm 13$ & $1237 \pm 20$ & 8 & 0.08164 & 1 & 2.168 & 1.6 & 0.1926 & 1.2 & 0.763 \\
\hline 47.1 & 0.11 & 691 & 461 & 0.69 & 97.7 & $982 \pm 11$ & $1224 \pm 17$ & 20 & 0.0811 & 0.86 & 1.84 & 1.5 & 0.1645 & 1.2 & 0.812 \\
\hline 48.1 & 0.08 & 543 & 402 & 0.76 & 122 & $1491 \pm 17$ & $1884 \pm 15$ & 21 & 0.11529 & 0.84 & 4.136 & 1.5 & 0.2602 & 1.3 & 0.834 \\
\hline 49.1 & 0.32 & 156 & 108 & 0.71 & 32.5 & $1393 \pm 26$ & $1590 \pm 47$ & 12 & 0.0982 & 2.5 & 3.27 & 3.2 & 0.2411 & 2 & 0.630 \\
\hline 50.1 & 0.26 & 215 & 69 & 0.33 & 36.1 & $1148 \pm 16$ & $1415 \pm 37$ & 19 & 0.0895 & 2 & 2.406 & 2.5 & 0.1949 & 1.5 & 0.619 \\
\hline 51.1 & 0.00 & 274 & 73 & 0.27 & 50.5 & $1252 \pm 16$ & $1587 \pm 22$ & 21 & 0.098 & 1.2 & 2.898 & 1.9 & 0.2144 & 1.4 & 0.765 \\
\hline 52.1 & 0.69 & 776 & 337 & 0.45 & 115 & $1017 \pm 10$ & $1351 \pm 19$ & 25 & 0.08656 & 0.98 & 2.039 & 1.5 & 0.1708 & 1.1 & 0.739 \\
\hline 53.1 & 0.03 & 83 & 87 & 1.08 & 25.3 & $1948 \pm 25$ & $1916 \pm 15$ & -2 & 0.11736 & 0.84 & 5.708 & 1.7 & 0.3527 & 1.5 & 0.867 \\
\hline
\end{tabular}




\begin{tabular}{|c|c|c|c|c|c|c|c|c|c|c|c|c|c|c|c|}
\hline \multicolumn{16}{|c|}{ ontinued } \\
\hline 54.1 & 0.43 & 559 & 93 & 0.17 & 89.6 & $1099 \pm 11$ & $1219 \pm 17$ & 10 & 0.08091 & 0.87 & 2.073 & 1.4 & 0.1858 & 1.1 & 0.781 \\
\hline 55.1 & 0.00 & 268 & 79 & 0.30 & 54.4 & $1365 \pm 14$ & $1349 \pm 12$ & -1 & 0.0865 & 0.64 & 2.813 & 1.3 & 0.2358 & 1.2 & 0.875 \\
\hline 56.1 & 0.00 & 182 & 50 & 0.28 & 43.5 & $1584 \pm 17$ & $1578 \pm 20$ & 0 & 0.0976 & 1.1 & 3.747 & 1.6 & 0.2785 & 1.2 & 0.750 \\
\hline 57.1 & 0.13 & 289 & 332 & 1.19 & 71.5 & $1629 \pm 16$ & $1779 \pm 12$ & 8 & 0.10879 & 0.66 & 4.313 & 1.3 & 0.2875 & 1.1 & 0.865 \\
\hline 58.1 & 0.09 & 216 & 233 & 1.12 & 56.2 & $1708 \pm 18$ & $1952 \pm 10$ & 13 & 0.11974 & 0.57 & 5.007 & 1.3 & 0.3033 & 1.2 & 0.901 \\
\hline 59.1 & 0.03 & 142 & 46 & 0.33 & 29.9 & $1411 \pm 16$ & $1345 \pm 17$ & -5 & 0.08629 & 0.88 & 2.911 & 1.6 & 0.2447 & 1.3 & 0.821 \\
\hline 60.1 & 0.01 & 237 & 255 & 1.11 & 98.6 & $2550 \pm 24$ & $2573.4 \pm 6.1$ & 1 & 0.17161 & 0.36 & 11.48 & 1.2 & 0.4853 & 1.2 & 0.954 \\
\hline 61.1 & 0.19 & 220 & 228 & 1.07 & 53.7 & $1613 \pm 17$ & $1793 \pm 12$ & 10 & 0.10959 & 0.63 & 4.297 & 1.3 & 0.2844 & 1.2 & 0.878 \\
\hline 62.1 & 0.22 & 507 & 170 & 0.35 & 118 & $1547 \pm 15$ & $1882.3 \pm 8.3$ & 18 & 0.11516 & 0.46 & 4.308 & 1.2 & 0.2713 & 1.1 & 0.919 \\
\hline 63.1 & 0.00 & 92 & 99 & 1.11 & 29.4 & $2043 \pm 24$ & $2019 \pm 12$ & -1 & 0.12434 & 0.68 & 6.394 & 1.5 & 0.373 & 1.4 & 0.897 \\
\hline 64.1 & 0.00 & 60 & 52 & 0.89 & 15.9 & $1740 \pm 24$ & $1724 \pm 19$ & -1 & 0.1055 & 1 & 4.508 & 1.9 & 0.3098 & 1.6 & 0.840 \\
\hline 65.1 & 0.03 & 304 & 55 & 0.19 & 52 & $1169 \pm 12$ & $1190 \pm 13$ & 2 & 0.07973 & 0.66 & 2.186 & 1.3 & 0.1988 & 1.2 & 0.867 \\
\hline 66.1 & 0.13 & 323 & 240 & 0.77 & 122 & $2336 \pm 22$ & $2696.8 \pm 5.6$ & 13 & 0.18484 & 0.34 & 11.13 & 1.2 & 0.4368 & 1.1 & 0.957 \\
\hline 67.1 & 0.00 & 206 & 125 & 0.63 & 48.1 & $1550 \pm 16$ & $1555 \pm 12$ & 0 & 0.09635 & 0.62 & 3.612 & 1.3 & 0.2719 & 1.2 & 0.887 \\
\hline 68.1 & 0.13 & 34 & 24 & 0.72 & 9.66 & $1842 \pm 29$ & $1826 \pm 25$ & -1 & 0.1116 & 1.4 & 5.09 & 2.3 & 0.3307 & 1.8 & 0.799 \\
\hline 69.1 & 0.04 & 192 & 141 & 0.76 & 94.6 & $2924 \pm 28$ & $3009.1 \pm 5.4$ & 3 & 0.22392 & 0.33 & 17.72 & 1.2 & 0.574 & 1.2 & 0.963 \\
\hline 70.1 & 0.82 & 614 & 713 & 1.20 & 92.4 & $1033 \pm 10$ & $1590 \pm 18$ & 35 & 0.09818 & 0.96 & 2.352 & 1.5 & 0.1737 & 1.1 & 0.751 \\
\hline
\end{tabular}

Errors are 1-sigma; $\mathrm{Pb}_{\mathrm{c}}$ and $\mathrm{Pb}^{*}$ indicate the common and radiogenic portions, respectively. Error in Standard calibration was $0.37 \%$ (not included in above errors but required when comparing data from different mounts). (1) Common $\mathrm{Pb}$ corrected using measured ${ }^{204} \mathrm{~Pb}$.

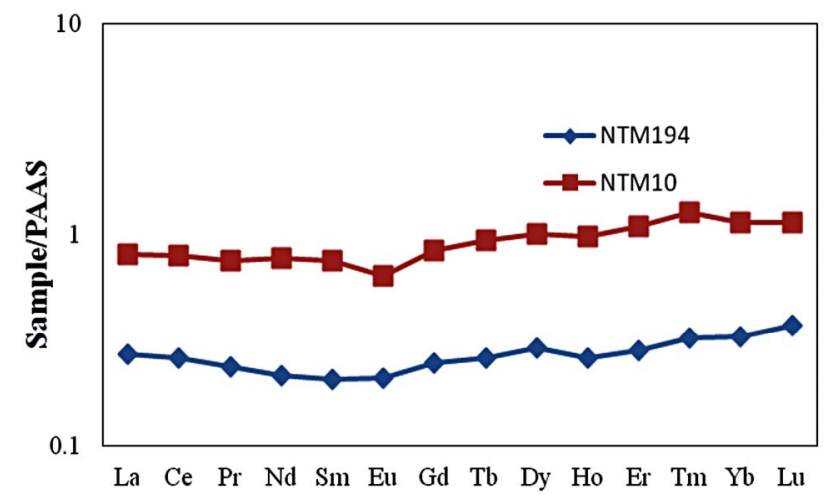

Figure 4. PAAS normalized REE spidergram for the metasandstone (NTM194) and metasiltstone (NTM10).

and metasiltstone with $\left(\mathrm{Eu} / \mathrm{Eu}^{*}\right)_{\mathrm{N}}$ values of 0.9248 and 0.7954 , respectively. The weak HREE enrichment pattern over the LREE and the flat Ce-anomaly is possibly explained by the dilution of the ocean sediments by riverine inputs [55]. The $\Sigma$ REE for the metasandstone and metasiltstone are $\sim 4 \mathrm{ppm}$ and $13 \mathrm{ppm}$, respectively. Generally, clastic sediments have $\Sigma$ REE in magnitude higher than 7. This is evident in the metasiltstone sample. Meanwhile, the poor REE concentration in the metasandstone may be attributed to its high maturity and the dilution effect of quartz given that REEs are chiefly supplied in sediments by the clay fraction. A similar explanation is attributed to the flat and weak negative Eu-anomalies of the metasandstone and metasiltstone samples, respectively.

\subsection{Detrital Zircon Geochronology}

The zircons from this sandstone are typical of sediments of this nature - small, rounded and highly variable in terms of their internal structures such as zoning and core/rim relationships. There are no overgrowths. Figure 5(a) is a SEM cathodoluminescence image of a representative selection of grains, showing the sites dated by SHRIMP. In order to get a statistically reliable picture of the provenance of the zircons in the sandstone, 70 different zircons were dated (see data in Table 2 and Figure 5(b)). There is a very wide range of ages from just over 3 Ga to a population with a grouping at about $1200 \mathrm{Ma}$. Most zircons are Palaeoproterozoic, but a significant proportion of the grains analyzed fall within the range 1600 - 1200 Ma. Figure 5(c) is a probability density plot of the more concordant data (i.e. the most reliable in terms of ages) showing the ages of the source rocks.

The maximum age of deposition of the sandstone is given by the youngest cluster of data, in this case at $\sim 1200$ Ma.

\section{DISCUSSION AND CONCLUSIONS}

Textural, mineralogical and whole rock geochemical data suggest that the metasediments of the Ntem complex range from mature to immature and the clastics are supplied from sources with various lithologic units. Evidence of diagenetic alteration and deformation in the sediments is provided by the recrystallization of quartz and the development of a tectonic fabric. Several geochronology studies have confirmed the Archean age of the Ntem Complex $[19,20,28,32,56]$.

Three zircon age groups are distinguished from the metasandstone sample (NTM194) investigated here and these are:

- Group A: Archean age reported on only few grains with ages of $2573.4 \pm 6.1 \mathrm{Ma}, 2696.8 \pm 5.6 \mathrm{Ma}$ and $3009.1 \pm 5.4 \mathrm{Ma}$. 


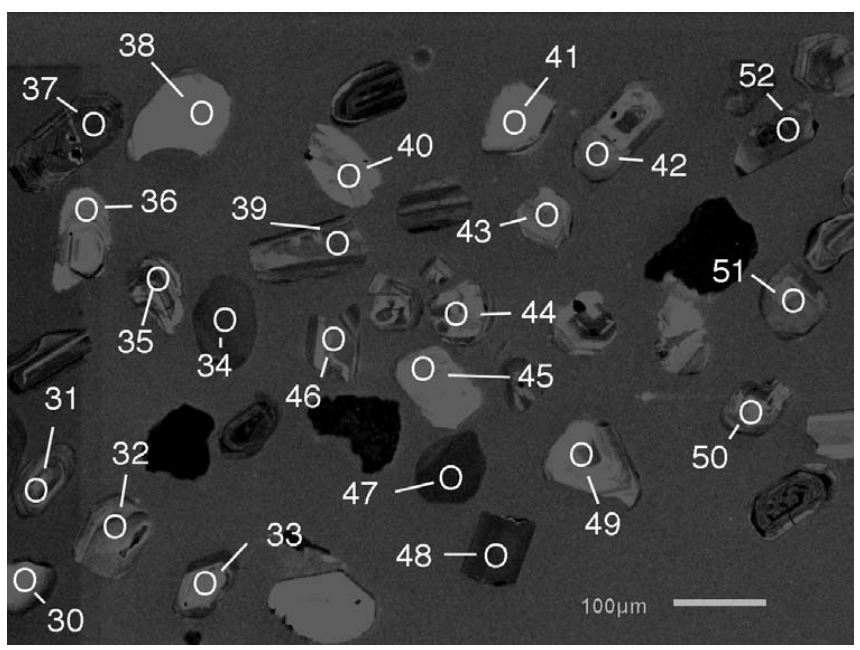

(a)

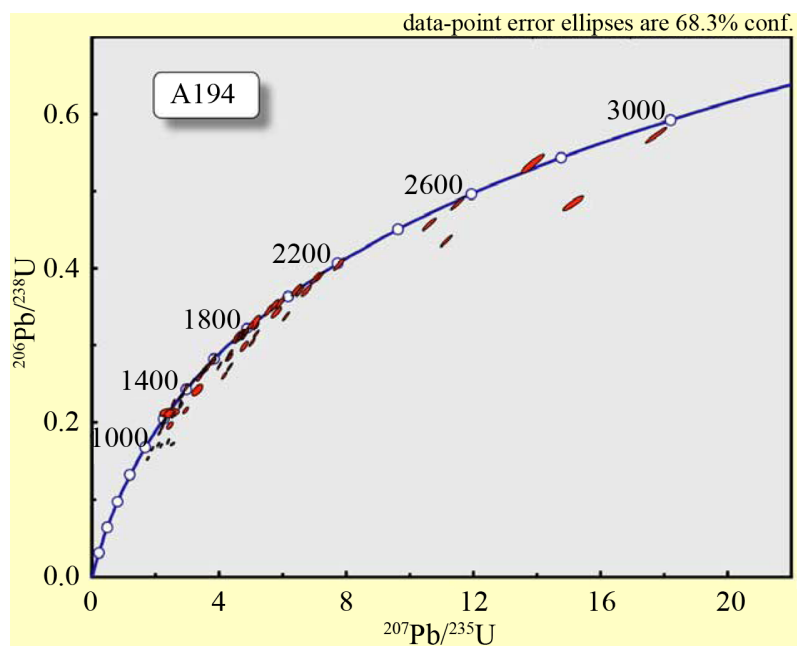

(b)

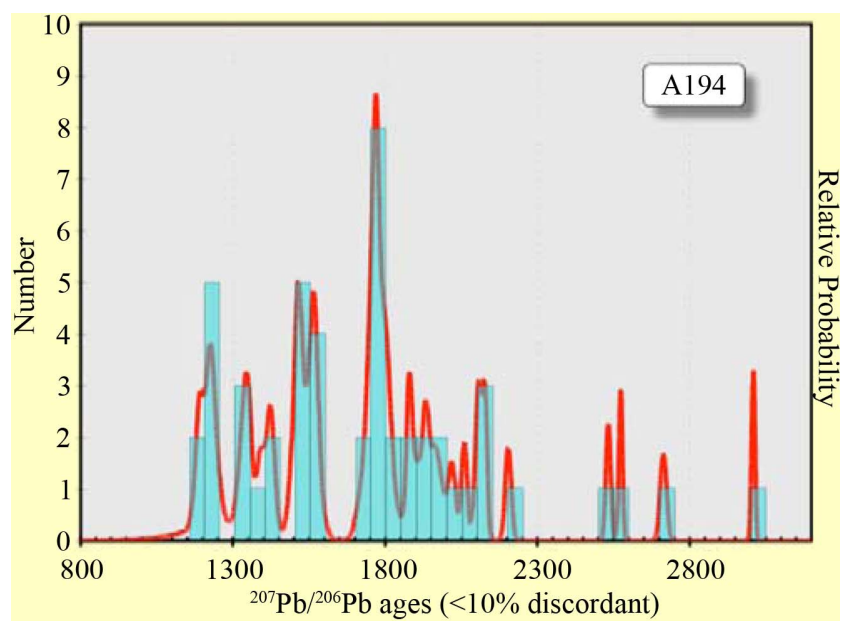

(c)

Figure 5. (a) Cathodoluminescence image of zircons separated and analysed from the sandstone sample NTM194. The numbered ellipses mark the spots analyzed; (b) U-Pb concordia plot of detrital zircon data from the sandstone NTM194 (c) A probability density plot of ${ }^{207} \mathrm{~Pb} /{ }^{206} \mathrm{~Pb}$ ages of detrital zircon data from the sandstone NTM194. Only the data that are $<10 \%$ discordant are considered for this assessment of the data.

- Group B: Paleoproterozoic age representing the dominant fraction with ages between 1700 and $2400 \mathrm{Ma}$.

- Group C: Mesoproterozoic to Neoproterozoic with ages between 1000 and $1600 \mathrm{Ma}$.

The variable age ranges reflect the different sources of sediments supplied into this sub-basin within the broader Congo basin. The Archean sources are interpreted as locally reworked zircons obtained from the alteration of the charnockitic assemblage and late Archean granitic intrusions within the basin. Some of the Archean ages are comparable to those obtained by [28] (2666 $\pm 2 \mathrm{Ma}$ and $2687 \pm 3 \mathrm{Ma})$ on the high-K granitoids of the Ntem Complex by $\mathrm{Pb}-\mathrm{Pb}$ zircon evaporation analysis. Also, [56] reported $\mathrm{Rb}-\mathrm{Sr}$ and $\mathrm{Sm}-\mathrm{Nd}$ whole rock data with ages around $2900 \mathrm{Ma}$ for the charnockitic suite and interprets this to define the timing of the granitic intrusion within the Ntem Complex. Using the same method, the authors later obtained $2721 \mathrm{Ma}$ for the high-K granitic group and suggest that the granitic suites are formed from the partial remelting of the charnockitic assemblage. A similar age of $2751 \pm 32 \mathrm{Ma}$ by U-Pb and $2719 \pm 9 \mathrm{Ma}$ as well as $2724 \pm 3 \mathrm{Ma}$ by $\mathrm{Pb}-\mathrm{Pb}$ evaporation is confirmed on zircons from the high-K granites by the same author 4 years later though variations in the dataset are largely attributed to $\mathrm{Pb}$ loss [20]. The slightly younger ages of $2573 \pm$ $6 \mathrm{Ma}$ and $2696 \pm 5 \mathrm{Ma}$ obtained for sample NTM194 in this study compared to the $2719 \pm 9 \mathrm{Ma}$ and $2724 \pm 3 \mathrm{Ma}$ obtained by [20] suggest the effect of post depositional processes on the zircons though the striking similarity with the ages reported by [57] who simply confirms his earlier interpretation that the magmatic episode postdates the main crust formation event by more than 200 Ma. Similarly, the $2573 \pm 6$ Ma age also compares with some younger ages of 2515 - 2566 Ma obtained by [58] 
using $\mathrm{U}-\mathrm{Pb}$ on detrital zircons from Late Archean metasedimentary rocks from the Wutai Complex, North China Craton where the authors attributed the zircon source to the well-defined calc-alkaline granitoids and the volcanic rocks in the Wutai Complex. The older age of $3009 \pm 5$ $\mathrm{Ma}$ is comparable to $\mathrm{U}-\mathrm{Pb}$ zircon ages of $3266 \pm 5 \mathrm{Ma}$, $3399 \pm 6 \mathrm{Ma}$ and $3477 \pm 16 \mathrm{Ma}$ reported by [19] on the charnockitic suite within the Ntem basin buttressing the locally derived source of the Archean zircon fraction.

The Group B (Paleoproterozoic) zircon ages are attributed to sources from the neighbouring Nyong Series west of the Ntem Complex. This unit has earlier been regarded as a reactivated northwest corner of the Congo Craton [59]. U-Pb zircons ages of $2300 \mathrm{Ma}$ have been reported from metasyenites north of Lolodorf within the Nyong Series [57]. More recently, [60] reports new SHRIMP ages of $2423 \pm 4 \mathrm{Ma}$ on detrital zircons from BIF, orthopyroxene gneisses and garnet gneisses of the Nyong Series. These ages are similar to most of the 2400 Ma to 1800 Ma Paleoproterozoic ages obtained for a significant population of the zircons analyzed in this study. [30] has also reported U-Pb ages of $2064 \mathrm{Ma}$ on zircons obtained from metamorphic rocks within the Nyong Series. This age bracket has also been reported $(2.0 \mathrm{Ga})$ by SHRIMP dating on detrital zircons from sandstones of the Gackowa Formation, Kaczawa Complex Sudetes, SW Poland by [61] and is interpreted to represent inherited components in the Variscan crust and provides evidence for intense crust-forming processes in Early Proterozoic time [62-64]. This arguably could also explain the dominant Paleoproterozoic age of the zircons analyzed in this study adding to the wider recognition of crustal growth in the Paleoproterozoic across the globe. It also largely points to the supply of clastics from the neighbouring Nyong Series into the Congo Basin buttressing the peak of deposition in the sub-basin during this period.

The younger Mesoproterozoic to Neoproterozoic ages are attributed to variable sources from the neighbouring units particularly from the West Congo Supergroup at the western edge of the Congo Craton and the mobile belts in Cameroon north of the Ntem complex. Zircons of similar ages have been obtained from sediments of the Poli, Lom and Yaoundé Series and this is eloquent proof that the Ntem sub-basin was still active during sedimentation in these pull-apart basins in north central Cameroon. [65] has earlier reported such Mesozoic to early Neoproterozoic ages of 1100 - 910 Ma from magmatic and sedimentary sequences of the Zadinian and Mayumbian Groups (Bas-Congo) west of the Congo Craton. A similar age of $1017 \mathrm{Ma}$ by U-Pb on detrital zircons was later reported by [66] from the Mahan amphiboites in the Yaoundé Series and was interpreted as the product of detritus from Neoproterozoic magmatic arcs and Paleo proterozoic igneous basement of the Adamawa-Yadé domain. The similarity of these ages with the Group $\mathrm{C}$ zircons of this study possibly explains their provenance. Such younger ages (990 Ma, $1200 \mathrm{Ma}, 1400 \mathrm{Ma}, 1540$ Ma) have been reported by [68] on detrital zircons in diamictites from the Puga Hill and are tied to a common source from the nearby Amazonian Craton. Based on the $\mathrm{U}-\mathrm{Pb}$ ages obtained, we conclude that the Congo basin opened up sometime in the Archean and remained active up till the Neoproterozoic with the maximum age of deposition around $1200 \mathrm{Ma}$ with a wide sediment influx from the neighbouring units. The BIF intercalated metasandstone sample dated clearly shows the contribution of clastic sediments from the continent to the formation of Banded Iron Formation in the Congo Basin. The age similarity of the Mesozoic to Neoproterozoic zircons from the metasandstone with ages reported from the Amazonian Craton further enhances the link between the Congo Craton and the São Francisco Craton in Pre-Gondwana time.

\section{ACKNOWLEDGEMENTS}

This paper is part of the Ph.D. thesis of Nelson Chombong at the University of Buea and undertaken within the collaboration framework between the University of Buea and TU Clausthal, Germany, sponsored by the Alexander von Humboldt Foundation (AvH Stiftung) and coordinated by Prof. Bernd Lehmann to whom we are most grateful. We are most thankful to R. Armstrong and M. Fanning of ANU laboratory for the zircon dating. Emmanuel C. Suh and Charles D. C. Ilouga acknowledge support from CamIron that enables the mapping and sampling of the Njweng prospect.

\section{REFERENCES}

[1] James, H.J. (1954) Sedimentary facies of iron formation. Economic Geology, 49, 235-293. doi:10.2113/gsecongeo.49.3.235

[2] Dorr II, J.V.N. (1964) Supergene iron ores of Minas Gerais, Brazil. Economic Geology, 59, 1203-1240. doi:10.2113/gsecongeo.59.7.1203

[3] Holland, H.D. (1973) Ocean-possible source of iron in iron formations. Economic Geology, 68, 1169-1172. doi:10.2113/gsecongeo.68.7.1169

[4] Jacobsen, S.B. and Pimentel-Klose, M.R. (1988) Nd isotopic variations in Precambrian banded iron formations. Geopysical Research Letters, 15, 393-396. doi:10.1029/GL015i004p00393

[5] Bau, M. and Möller, P. (1993) Rare earth element systematics of the chemical precipitated component in Early Precambrian iron formations and the evolution of the terrestrial atmosphere-hydrosphere system. Geochimica et Cosmochemica Acta, 57, 2239-2249. doi:10.1016/0016-7037(93)90566-F

[6] Isley, A.E. (1995) Hydrothermal plumes and the delivery of iron to banded iron formations. Journal of Geology, 
103, 169-185. doi:10.1086/629734

[7] Beukes, N.J., Dorland, H.D., Gutzmer, J., Nedachi, M. and Ohmoto, H. (2002) Tropical laterites, life on land and the history of atmospheric oxygen in the Paleoproterozoic. Geology, 30, 419-494. doi:10.1130/0091-7613(2002)030<0491:TLLOLA $>2.0 . \mathrm{C}$ $\underline{\mathrm{O} ; 2}$

[8] Clout, J.M.F. and Simonson, B.M. (2005) Precambrian iron formations and iron formations-hosted iron ore deposits. Economic Geology, 100, 643-679.

[9] Beukes, N.J. and Gutzmer, J. (2008) Origin and paleoenvironmental significance of major iron formations of the archean-paleoproterozoic boundary. Reviews in Economic Geology, 15, 5-47.

[10] Steinhoefel, G., Von Blanckenburg, F., Horn, I., Konhauser, K.O., Beukes, N.J. and Gutzmer, J. (2010) Deciphering formation processes of banded iron formations from the Transvaal and the Hamersley successions by combined $\mathrm{Si}$ and $\mathrm{Fe}$ isotope analysis using UV femtosecond laser ablation. Geochimica et Cosmochimica Acta, 74, 2677-2696. doi:10.1016/i.gca.2010.01.028

[11] Garland, F.S., Turner, S. and Hawkesworth, C. (1996) Shifts in the source of the Parana basalts through time. Lithos, 37, 223-243. doi:10.1016/0024-4937(95)00038-0

[12] Isley, A.E. and Abbott, D.H. (1999). Plume-related mafic volcanism and the deposition of banded iron formation. Geophysical Research, 104, 15461-15477. doi:10.1029/1999JB900066

[13] Campbell, L.H., Griffiths, R.W. and Hill, R.I. (1989) Melting in an Archean mantle plume: Heads its basalts, tails its komatiites. Nature, 339, 697-699. doi: $10.1038 / 339697 \mathrm{a} 0$

[14] Richards, M.A., Duncan, R.A. and Comtillot, V.E. (1989) Flood basalts and hot-spot tracks: Plume heads and tails. Science, 246, 103-107. doi:10.1126/science.246.4926.103

[15] Robert, F. and Chaussidon, M. (2006) A palaeotemperature curve for the Precambrian oceans based on silicon isotopes in cherts. Nature, 443, 969-972. doi:10.1038/nature05239

[16] Van den Boorn, S.H.J.M., Van Bergen, M.J., Nijman, W. and Vroon, P.Z. (2007) Dual role of sea water and hydrothermal fluids in Early Archean chert formation: Evidence from silicon isotopes. Geology, 35, 939-942. doi:10.1130/G24096A.1

[17] Van den Boorn, S.H.J.M., Van Bergen, M.J., Vroon, P.Z., Vries, S.T. and Nijman. W. (2010) Silicon isotopes and trace elements constraints on the origin of $\sim 3.5 \mathrm{Ga}$ cherts: Implications for early Archean marine environments. Geochimica et Cosmochimica Acta, 74, 1077-1103. doi:10.1016/j.gca.2009.09.009

[18] Simonson, B.M. (2003) Origin and evolution of large Precambrian iron formations. Geological Society of America Special Paper, 370, 231-244.

[19] Takam, T., Arima, M., Kokonyangi, J., Dunkley, D.J. and Nsifa, E.N. (2009) Paleoarchean charnockite in the Ntem Comples, Congo Craton, Cameroon: Insights from SHRIMP zircon U-Pb ages. Journal of Mineralogical and
Petrological Sciences, 104, 1-11. doi: $10.2465 / \mathrm{jmps} .080624$

[20] Shang, C.K., Liégeois, J.P., Satir, M., Frisch, W. and Nsifa, E.N. (2010). Late Archaean high-K granite geochronology of the northern metacratonic margin of the Archaean Congo craton, Southern Cameroon: Evidence for $\mathrm{Pb}$-loss due to non-metamorphic causes. Gondwana Research, 18, 337-355. doi:10.1016/j.gr.2010.02.008

[21] Pouclet, A., Tchameni, R., Mezger, K., Vidal, M., Nsifa, E.N., Shang, C.K. and Penaye, J. (2007) Archean crustal accretion at the northern border of the Congo craton (South Cameroon). The charnockite-TTG link. Bulletin of the Geological Society of France, 178, 331-342. doi:10.2113/gssgfbull.178.5.331

[22] Shang, C.K., Satir, M., Nsifa, E.N., Liegeois, J.P., Siebel, W. and Taubald, H. (2007). Archean high K-granitoids produced by remelting of early Tonalite-TrondhjemiteGranodiorite (TTG) in the Sangmelima region of the Ntem Complex of the Congo craton, southern Cameroon. International Journal of Earth Sciences, 96, 817-842. doi:10.1007/s00531-006-0141-3

[23] Suh, C.E., Cabral, A.R., Shemang, E.M., Mbinkar, L. and Mboudou, G.G.M. (2008) Two contrasting iron-ore deposits in the Precambrian mineral belt of Cameroon, West Africa. Exploration and Mining Geology, 17, 197-207. doi:10.2113/gsemg.17.3-4.197

[24] Suh, C.E., Cabral, A.R. and Ndime, E. (2009) Geology and ore fabrics of the Nkout high-grade haematite deposit, southern Cameroon. In: Williams, P.J., et al., Eds., Smart Science for Exploration and Mining, SGA Publication series, Amsterdam, 558-560.

[25] Nforba, M.T., Suh, C.E. and Kabeyene, K.V.K. (2010) Mbalam iron ore project, northern edge of the Congo craton, southeast Cameroon. In: Goldfarb, R.J., Marsh, E.E. and Monecke, E., Eds., Proceedings of the Society of Economic Geologists on the Challenge of Finding New Mineral Resources: Global Metallogeny, Innovative Exploration and New Discoveries, SEG Extended Abstracts, Colorado, G-22.

[26] Ilouga, C.D.I., Suh, C.E. and Ghogomu, R.T. (2013) Textures and rare earth elements composition of Banded Iron Formations (BIF) at Njweng prospect, Mbalam Iron Ore District, Southern Cameroon. International Journal of Geosciences, 4, 146-165. doi:10.4236/ijg.2013.41014

[27] Milesi, J.P., Toteu, S.F., Deschamps, Y., Feybesse, J.L., Lerouge, C., Cocherie, A., Penaye, J., Tchameni, R., Moloto-A-Kenguemba, G., Kampunzu, H.A.B., Nicol, N., Duguey, E., Leistel, J.M., Saint-Martin, M., Ralay, F., Heinry, C., Bouchot, V., Doumnang Mbaigane, J.C., Kanda Kula, V., Chene, F., Monthel, M., Boutin, B. and Cailteux, J. (2006) An overview of the geology and major ore deposits of Central Africa: Explanatory note for the 1: 4000,000 map "Geology and major ore deposits of Central Africa”. Journal of African Earth Sciences, 44, 571595. doi:10.1016/j.jafrearsci.2005.10.016

[28] Tchameni, R., Mezger, K., Nsifa, N.E. and Pouclet, A. (2000) Neoarchean evolution of the Congo craton: Evidence from K-rich granitoids of the Ntem Complex, southern Cameroon. Journal of African Earth Sciences, 30, 133-147. doi:10.1016/S0899-5362(00)00012-9 
[29] Feybesse, J.L., Johan, V., Triboulet, C., Guerrot, C., Mayaga-Mikolo, F., Bouchot, V. and Ekondong, J. (1998) The West Central African belt: A model of 2.5-2.0Ga accretion and two-phase orogenic evolution. Precambrian Research, 87, 161-216. doi:10.1016/S0301-9268(97)00053-3

[30] Shang, C.K., Satir, M., Siebel, W., Nsifa, E.N., Taubald, H., Liégeois, J.P. and Tchoua, F.M. (2004) TTG magmatism in the Congo craton: Case study of the Sangmalima region, Ntem Complex, southern Cameroon. Journal of African Earth Sciences, 40, 61-79. doi:10.1016/i.jafrearsci.2004.07.005

[31] Alkmim, F.F., Marshak, S., Pedrosa-Soares, A.C., Peres, G.G., Cruz, S.C.P. and Whittington, A. (2006) Kinematic evolution of the Araçuaí-West Congo orogeny in Brazil and Africa: Nutcracker tectonics during the Neoproterozoic assembly of Gondwana. Precambrian Research, 149, 43-46. doi:10.1016/j.precamres.2006.06.007

[32] Van Schmus, W.R., Oliveira, E.P., da Silva Filho, E.F., Toteu, S.F., Penaye, J. and Guimarães, I.P. (2008) Proterozoic links between the Borborema Province, NE Brazil, and the Central African Fold Belt. Geological Society, Special Publications, London, 294, 69-99.

[33] Danderfer, A., De Waele, B., Pedreira, A.J. and Nalini, H.A. (2009) New geochronological constraints on the geological evolution of Espinhaço basin within the São Francisco Craton-Brazil. Precambrian Research, 170, 116128. doi:10.1016/j.precamres.2009.01.002

[34] Neves, S.P., Bruguier, O., da Silva, J.M.R., Bosch, D., Alcantara, V.C. and Lima, C.M. (2009) The age distributions of detrital zircons in metasedimentary sequences in eastern Borborema Province (NE Brazil): Evidence for intercontinental sedimentation and orogenesis? Precambrian Research, 175, 187-205. doi:10.1016/j.precamres.2009.09.009

[35] Hollanda, M.H.B.M., Archanjo, C.J., Souza, L.C., Armstrong, R. and Vasconcelos, P.M. (2010) Cambrian mafic to felsic magmatism and its connections with transcurrent shear zones of the Borborema Province (NE Brazil): Implications for late assembly of West Gondwana. Precambrian Research, 178, 1-14.

doi:10.1016/j.precamres.2009.12.004

[36] Oliveira, E.P., Windley, B.F. and Araújo, M.N.C. (2010) The Neoproterozoic Sergipano orogenic belt, NE Brazil: A complete plate tectonic cycle in western Gondwana. Precambrian Research, 181, 64-84. doi:10.1016/j.precamres.2010.05.014

[37] Bueno, J.F., Oliveira, E.P., McNaughton, N. and Laux, J.H. (2009) U-Pb dating of granites in the Neoproterozoic Sergipano Belt, NE Brazil: Implications for the timing and duration of continental collision and extension tectonic in the Borborema Province. Gondwana Research, 15, 86-97. doi:10.1016/j.gr.2008.06.003

[38] Oliveira, E.P., Toteu, S.F., Araújo, M.N.C., Carvalho, M.J., Nascimento, R.S., Bueno, J.F., MCNaughton, N. and Basilici, G. (2006) Geologic correlation between the Neoproterozoic Sergipano belt (NE Brazil) and the Yaoundé Schists belt (Cameroon, Africa). Journal of African Earth Sciences, 44, 470-478.

doi:10.1016/j.jafrearsci.2005.11.014
[39] Chombong, N.N. and Suh, C.E. (2013) 2883 Ma commencement of BIF deposition at the northern edge of Congo craton, southern Cameroon: New zircon SHRIMP data constraint from metavolcanics. Episodes, 36, 47-57.

[40] Black, L.P., Kamo, S.L., Allen, C.M., Davis, D.W., Aleinikoff, J.N., Valley, J.W., Mundil, R., Campbell, I.H., Korch, R.J., Williams, L.S. and Foudoulis, C. (2004) Improved ${ }^{206} \mathrm{~Pb} /{ }^{238} \mathrm{U}$ microprobe geochronology by monitoring of a trace-element-related matrix effect; SHRIMP, ID-TIMS, ELA-ICP-MS and oxygen isotope documentation for a series of zircon standards. Chemical Geology, 205, 115-140. doi:10.1016/j.chemgeo.2004.01.003

[41] Paces, J.B. and Miller, J.D. (1993) Precise U-Pb age of Duluth Complex and related mafic intrusions, northeastern Minnesota: Geochronological insights to physical, petrogenetic, paleomagnetic, and tectonomagnetic processes associated with the $1.1 \mathrm{Ga}$ Midcontinent rift system. Journal of Geophysical Research, 98, 13997-14013. doi:10.1029/93JB01159

[42] Claoué-Long, J.C., Compston, W., Roberts, J. and Fanning, C.M. (1995) Two Carboniferous ages: A compareson of SHRIMP zircon dating with conventional zircon ages and 40Ar/39Ar analysis. In: Berggen, W.A., Kent, D.V., Aubry, M.P. and Hardenbol, J., Eds., Geochronology, Time Scales and Global Stratigraphic Correlation, SEPM Special Publication, Colorado, 3-21.

[43] Williams, I.S. (1998) U-Th-Pb geochronology by ion microprobe. In: McKibben, M.A., Shanks III, W.C. and Ridley, W.I., Eds., Applications of Microanalytical Techniques to Understanding Mineralising Processes, Society of Economic Geologists, Colorado, 1-35.

[44] Ludwig, K.R. (2001) SQUID 1.03, a user's manual. Berkeley Geochronology Center, Berkeley, 19.

[45] Steiger, R.H. and Jäger, E. (1977) Subcommision on geochronology: Convention on the use of decay constants in geo- and cosmochronology. Earth Planetary Science Letters, 36, 359-362. doi:10.1016/0012-821X(77)90060-7

[46] Stacey, J.S. and Kramers J.D. (1975) Approximation of terrestrial lead isotope evolution by a two-stage model. Earth Planetary Science Letters, 26, 207-221. doi:10.1016/0012-821X(75)90088-6

[47] Ludwig, K.R. (2003) Isoplot 3.00: A geochronological toolkit for Microsoft Excel. Berkeley Geochronology Center, Berkeley, 70.

[48] Borradaile, G.J., Bayly, M.B. and Powell, C.M.A. (1982) Atlas of deformational and metamorphic rock fabrics. Springer-Verlag, Berlin, 551. doi:10.1007/978-3-642-68432-6

[49] Wright, P.L. (1974) The chemistry and mineralogy of the clay fraction of sediments from the southern Barents Sea. Chemical Geology, 13, 197-216. doi:10.1016/0009-2541(74)90020-5

[50] McLennan, S.M. (1982) On the geochemical evolution of sedimentary rocks. Chemical Geology, 37, 335-350. doi:10.1016/0009-2541(82)90087-0

[51] McLennan, S.M. and Taylor, S.R. (1984) Archean sedimentary rocks and their relation to the composition of the Archean crust. In: Kroner, A., Ed., Archean Geochemistry, Springer-Verlag, Berlin, 47-72. 
doi:10.1007/978-3-642-70001-9 3

[52] Roser, B.P., Cooper, R.A., Nathan, S. and Tulloch, A.J. (1996) Reconnaissance sandstone geochemistry, provenance, and tectonic setting of the lower Paleozoic terranes of the West Coast and Nelson, New Zealand. New Zealand Journal of Geology and Geophysics, 39, 1-16. doi:10.1080/00288306.1996.9514690

[53] Brynton, W.V. (1984) Geochemistry of the rare earth elements: Meteorite studies. In: Henderson, P., Ed., Rare Earth Elements Petrochemistry, Elsevier, Amsterdam, 63-114.

[54] Derry, L.A. and Jacobsen, S.B. (1990) The chemical evolution of Precambrian seawater: Evidence from REEs in banded iron formations. Geochimica et Cosmochimica Acta, 54, 2965-2977. doi:10.1016/0016-7037(90)90114-Z

[55] Elderfield, H., Upstill-Goddard, R. and Sholkovitz, E. R. (1990) The rare earth elements in rivers, estuaries, and coastal seas and their significance to the composition of ocean waters. Geochimica et Cosmochimica Acta, 54, 971-991. doi:10.1016/0016-7037(90)90432-K

[56] Shang, C.K., Siebel, W., Satir, M., Chen, F. and Mvondo, J.O. (2004) Zircon Pb-Pb and U-Pb systematic of TTG rocks in the Congo craton: Constraints on crust formation, magmatism and Pan-African lead loss. Bulletin of Geoscience, 79, 205-219.

[57] Tchameni, R., Mezger, K., Nsifa, N.E. and Pouclet, A. (2001) Crustal origin of early Proterozoic syenites in the Congo craton (Ntem Complex), southern Cameroon. Lithos, 57, 23-42. doi:10.1016/S0024-4937(00)00072-4

[58] Li, Q., Liu, S., Wang, Z., Chu, Z., Song, B., Wang, Y. and Wang, T. (2008) Contrasting provenance of Late Archean metasedimentary rocks from the Wutai Complex, North China Craton: Detrital zircon U-Pb, whole-rock Sm-Nd isotopic and geochemical data. International Journal of Earth Sciences (Geologische Rundschau), 97, 443-458. doi:10.1007/s00531-007-0170-6

[59] Lasserre, M. and Soba, D. (1976) Age Libérien des granodiorite et des gneiss à pyroxenes du Cameroun méridional. Bulletin of the Geological and Mining Research Department (BRGM), 2, 17-32.

[60] Lerouge, C., Cocherie, A., Toteu, S.F., Penaye, J., Milési, J.P., Tchameni, R., Nsifa, E.N., Fanning, C.M. and Doloule, E. (2006) Shrimp U-Pb zircon age evidence for Paleoproterozoic sedimentation and 2.05Ga syntectonic plutonism in the Nyong Group, SouthWestern Cameroon: Consequences for the Eburnean-Transamazonian belt of NE Brazil and Central Africa. Journal of African Earth Sciences, 44, 413-427. doi:10.1016/j.jafrearsci.2005.11.010
[61] Kryza, R., Zalasiewicz, J., Mazur, S., Aleksandrowski, P., Sergeev, S. and Larionov, A. (2007) Precambrian crustal contribution to the Variscan accretionary prism of the Kaczawa Mountains (Sudetes, SW Poland): Evidence from SHRIMP dating of detrital zircons. International Journal of Earth Sciences (Geologische Rundschau), 96, 1153-1162. doi:10.1007/s00531-006-0147-x

[62] Gebauer, D., Williams, I.S., CompstoN, W. and Grünenfelder, M. (1989) The development of the central European continental crust since the early Archean based on conventional and ion-microprobe dating of up to 3.84 by old detrital zircons. Tectonopysics, 157, 81-96. doi:10.1016/0040-1951(89)90342-9

[63] Friedl, G., Finger, F., McNaughton, N.J. and Fletcher, I.R. (2000) Deducing the ancestry of terranes: SHRIMP evidence for South America-derived Gondwana fragments in central Europe. Geology, 28, 1035-1038. doi:10.1130/0091-7613(2000)28<1035:DTAOTS $>2.0$.CO ;2

[64] Friedl, G., Finger, F., Paquette, J.L., von Quadt, A., McNaughton, N.J. and Fletcher, I.R. (2004) Pre-Variscan geological events in the Austrain part of the Bohemian Massif deduced from U-Pb zircon ages. International Journal of Earth Sciences (Geologische Rundschau), 93, 802-823. doi:10.1007/s00531-004-0420-9

[65] Tack, L., Wingate, M.T.D., Liégeois, J.P., FernadezAlonso, M. and Deblond, A. (2001). Early Neoproterozoic magmatism (1000-910Ma) of the Zadinian and Mayumbian Groups (Bas-Congo): Onset of Rodinia rifting at the western edge of the Congo Craton. Precambrian Research, 110, 277-306. doi:10.1016/S0301-9268(01)00192-9

[66] Toteu, S.F., Penaye, J., Deloule, E., Van Schmus, W.R. and Tchameni, R. (2006) Diachronous evolution of volcano-sedimentary basins north of the Congo craton: Insights from $\mathrm{U}-\mathrm{Pb}$ ion microprobe dating of zircons from the Poli, Lom and Yaounde Groups (Cameroon). Journal of African Earth Sciences, 44, 428-442. doi:10.1016/j.jafrearsci.2005.11.011

[67] Maurizot, P., Abessolo, A., Feybesse, J.L., Johan, V. and Lecomte, P. (1986) Etude et prospection minière du Sud-Ouest Cameroon. Synthèse des travaux de 1978 à 1985. Geological and Mining Research Department (BRGM) Rreport, 85, 66.

[68] Babinski, M., Boggiani, P.C., Trindade, R.I.F and Fanning, C.M. (2012) Detrital zircon ages and geochronological constraints on the Neoproterozoic Puga diamictites and associated BIFs in the southern Paraguay Belt, Brazil. Gondwana Research, 3, 988-997. 